\title{
Polymers in fluid flows
}

\author{
Roberto Benzi ${ }^{1}$ and Emily S.C. Ching ${ }^{2}$ \\ ${ }^{1}$ Department of Physics, Univ. Roma "Tor Vergata"; email: benzi@roma2.infn.it and \\ ${ }^{2}$ Department of Physics, The Chinese University of Hong Kong, \\ Shatin, Hong Kong; email: ching@phy.cuhk.edu.hk
}

(Dated: October 29, 2018)

\begin{abstract}
The interaction of flexible polymers with fluid flows leads to a number of intriguing phenomena observed in laboratory experiments, namely drag reduction, elastic turbulence and heat transport modification in natural convection, and is one of the most challenging subjects in soft matter physics. In this paper we review our present knowledge on the subject. Our present knowledge is mostly based on direct numerical simulations performed in the last twenty years, which have successfully explained, at least qualitatively, most of the experimental results. Our goal is to disentangle as much as possible the basic mechanisms acting in the system in order to capture the basic features underlying different theoretical approaches and explanations.
\end{abstract}

Keywords: polymer-flow interaction, drag reduction, elastic turbulence, heat transport modification

PACS numbers:

Posted with permission from the Annual Review of Condensed Matter Physics, Volume 9 @ 2018 by Annual Reviews, http://www.annualreviews.org/

\section{INTRODUCTION}

The interaction of flexible polymers with fluid flows is one of the most challenging subjects in soft matter physics. Laboratory experiments show that even a minute concentration of polymers can dramatically change properties (drag reduction) of turbulent flows or, if the flow is laminar, can trigger a new form of turbulence named elastic turbulence. How to properly describe flow-polymer interactions and how to understand the basic physical phenomena observed in experiments is both a fundamental scientific issue and an important challenge to develop many industrial applications. In this paper we review our present knowledge on the subject. In the last twenty years, direct numerical simulations of polymer-flow dynamics have successfully explained, at least qualitatively, most of the experimental results. Starting from this consideration, our goal is to disentangle as much as possible the basic mechanism acting in the system. Many different theories have been proposed and we feel confident that future work can provide a reasonably unified approach on the subject.

\section{POLYMER FLOW INTERACTION}

\section{A. Polymer passive advection}

We start our review by understanding the dynamics of polymers advected by a turbulent fluid flow. A flexible polymer can be considered as a chain of $N \gg 1$ monomers. The size of a polymer in its stretched configuration is of the order of tens of micrometers. In most cases, this size is much smaller or at most equal to the smallest dissipative scale of turbulence, namely the Kolmogorov scale $\eta=\left(\nu^{3} / \epsilon\right)^{1 / 4}$, where $\nu$ is the kinematic viscosity of the fluid and $\epsilon$ is the rate of turbulent energy dissipation. In this section we consider a polymer to be a passive object advected by the flow velocity and neglect its feedback to the flow. The important information comes from the end-to-end vector $\mathbf{R}$ of the polymer configuration. In its simplest form the Lagrangian dynamics of $\mathbf{R}$ is described by [1-3]

$$
\partial_{t} R_{i}=-\frac{1}{2 \tau} f(R) R_{i}+R_{j} \partial_{j} u_{i}+\sqrt{\frac{R_{0}^{2}}{\tau}} w_{i}(t)
$$

where $u_{i}$ is the component of the velocity field, $\tau$ is the polymer relaxation time, $R_{0}^{2}$ is a parameter that takes into account the role of thermal fluctuations, and $w_{i}(t)$ is independent white noise, delta-correlated in time. The relaxation time $\tau$ depends on the chemical and physical properties of the polymer, and ranges from $10^{-3} \mathrm{~s}$ up to tens of seconds. The function $f(R)$ takes into account the finite extensibility of the polymer and that the maximum extension of polymer size $R=|\mathbf{R}|$ is $R_{\max }$. Hereafter we choose $f(R)=1 /\left(1-\alpha R^{2}\right)$, with $\alpha=1 / R_{\max }^{2}$. Using Equation 1 , we 
can describe the statistical properties of polymers by considering the conformational tensor $R_{i j}=\left\langle R_{i} R_{j}\right\rangle_{w}$ where the average is done on the noise. Upon using Ito calculus, the Eulerian equations for the conformational tensor are:

$$
\frac{d R_{i j}}{d t} \equiv \partial_{t} R_{i j}+u_{k} \partial_{k} R_{i j}=-\frac{1}{\tau}\left[f(R) R_{i j}-\delta_{i j} R_{0}^{2}\right]+R_{i k} \partial_{k} u_{j}+R_{j k} \partial_{k} u_{j}
$$

First we consider the case $\alpha=0$, assuming that $R<R_{\max }$ at any time. If we follow the polymer along its trajectory, the quantities $s_{i j} \equiv \partial_{j} u_{i}$ are a function of $t$ only. Starting from Equation 1 we are interested in computing the statistical properties of $R$. Following References [3 [5], we can compute the probability distribution $P(R)$ from the knowledge of the long time behavior of the Lyapunov exponent of the Lagrangian trajectory. Let $\mathbf{l}(t)$ be the solution of the linear equation:

$$
\frac{d l_{i}}{d t}=s_{i j} l_{j}
$$

with initial condition $|\mathbf{l}(0)|=1$. For chaotic flows, the size $l(t)=|\mathbf{l}(t)|$ grows exponentially in time as $\exp (\gamma t)$ where $\gamma$ is the finite time Lyapunov exponent, i,e, $\lim _{t \rightarrow \infty} \gamma=\lambda, \lambda$ being the Lyapunov exponent of the flow. It is also known [6] that the probability $P(\gamma)$ of observing the value $\gamma$ at time $t$ is given by $\exp [-t S(\gamma-\lambda)]$, where $S(x)$ is known as the Cramer entropy and is a convex function with a minimum at $x=0$. The Cramer entropy can be used to estimate the large deviation properties of $l(t)$. More precisely, one can show that $\left\langle l^{q}(t)\right\rangle=\exp [t L(q)]$ where $L(q)$ is the Legendre transform of $S(x)$, i.e. $L(q)=\sup _{x}[q x-S(x)]$. Asymptotically, we can estimate from Equation 1 that $R \sim \exp (-t / \tau+\gamma t)$ and that stretching properties of the polymer depend on the dimensionless number $\mathrm{Wi}_{\lambda} \equiv \lambda \tau$. The quantity $\mathrm{Wi}_{\lambda}$ is called Weissenberg number, although in many papers no reference to the Lyapunov exponent is made. Clearly there exists a critical value $\mathrm{Wi}_{\lambda, c}$ of $\mathrm{Wi}_{\lambda}$ for which the polymer tends to stretch indefinitely (for the case of $\alpha=0$ ). This critical value signals the so-called coil-stretch transition for the polymer. It is possible to show [3, 4] that, for $\mathrm{Wi}_{\lambda}<\mathrm{Wi}_{\lambda, c}$, the polymer extension $R$ shows a power law distribution $P(R) \sim R^{-1-a}$ where $a=2 \tau L(a)$. The quantity $L(q)$ is not known in general and depends on the intermittent features (if any) of the Lagrangian trajectory. In most cases one can assume as a first approximation $L(q)=\lambda q+\Delta q^{2} / 2$ and obtain

$$
a=\frac{\lambda}{\Delta}\left[\frac{1}{\mathrm{Wi}_{\lambda}}-2\right]
$$

From Equation 4 we can immediately see that $\mathrm{Wi}_{\lambda, c}=1 / 2$ : For $\mathrm{Wi}_{\lambda}<\mathrm{Wi}_{\lambda, c}$ the value of $a$ is positive and the probability distribution $P(R)$ is normalizable; for $\mathrm{Wi}_{\lambda}>\mathrm{Wi}_{\lambda, c}$ the exponent $a$ becomes negative and $P(R)$ is no longer normalizable. The ratio $\lambda / \Delta$ is a quantitative measure of intermittency for the Lagrangian trajectories: Strong intermittency implies $\lambda / \Delta \ll 1$ and small values of $a$; small intermittency is equivalent to $\lambda / \Delta \gg 1$ and large values of $a$.

For a large value of $\mathrm{Wi}_{\lambda}$, we must consider the case $\alpha>0$ in Equation 1 In this case, the analysis, performed in [8] and 9] in some simplified case, shows that coil-stretch transition does not change qualitatively, although the existence of a scaling range for $P(R)$ depends on flows details. The overall message coming from the above discussion is rather clear: A single polymer passively advected in a chaotic flow undergoes a coil-stretch transition when $\mathrm{Wi}_{\lambda}$ is larger than a critical value $\mathrm{Wi}_{\lambda, c}$. The precise value of $\mathrm{Wi}_{\lambda, c}$ depends on the flow properties [i.e. the function $L(q)$ ], although it is reasonable to guess it is of order one. So far one has not been able to measure $\lambda$ experimentally. For fully developed turbulence, one may use the simple estimate $\lambda \sim 1 / \tau_{\eta}$ where $\tau_{\eta}=\sqrt{\nu / \epsilon}$ is the Kolmogorov time. Alternatively, one can estimate $\lambda$ as $U / L$ where $U$ is a characteristic velocity of the flow and $L$ its characteristic scale. Quite often the Weissenberg number defined by $\mathrm{Wi}=U \tau / L$ is also referred to as the Deborah number, De. Not surprisingly, depending on the definition, the critical value of Wi or De changes considerably. However, the basic point of the above analysis is that the time criterion based on $\mathrm{Wi}_{\lambda}>\mathrm{Wi}_{\lambda, c}$ is the one to be used to capture the polymer coil-stretch transition.

$P(R)$ and coil-stretch transition have been investigated numerically [7, 10, 11] and experimentally in Reference [12] (see Figure 11). In the experiment, polymers were advected in a swirling flow between the edge of a uniformly rotating glass of radius $r_{1}$ and angular velocity $\Omega$. The Weissenberg number is defined as $\mathrm{Wi}=\tau \Omega r_{1} / d$ where $d$ is the gap between plates. The authors considered polymers with $R_{0} \sim 1 \mu \mathrm{m}$ and $R_{\max } \sim 20 \mu \mathrm{m}$ and they were able to measure the value $R$ by fluorescent techniques. A clear coil-stretch transition is observed for $\mathrm{Wi}=\mathrm{Wi}_{c} \sim 6$. For $\mathrm{Wi}<\mathrm{Wi}_{c}, P(R)$ shows a power-law distribution, and $a \sim 1.5$ for the smallest Wi studied. The experiment shows a rather remarkable qualitative agreement with the theoretical framework discussed in this section.

\section{B. When turbulence is affected by polymers}

After discussing how polymers are affected by a fluid flow, we now discuss the change in the fluid flow due to the presence of polymers. We consider three-dimensional homogeneous and isotropic turbulent flows far away from 


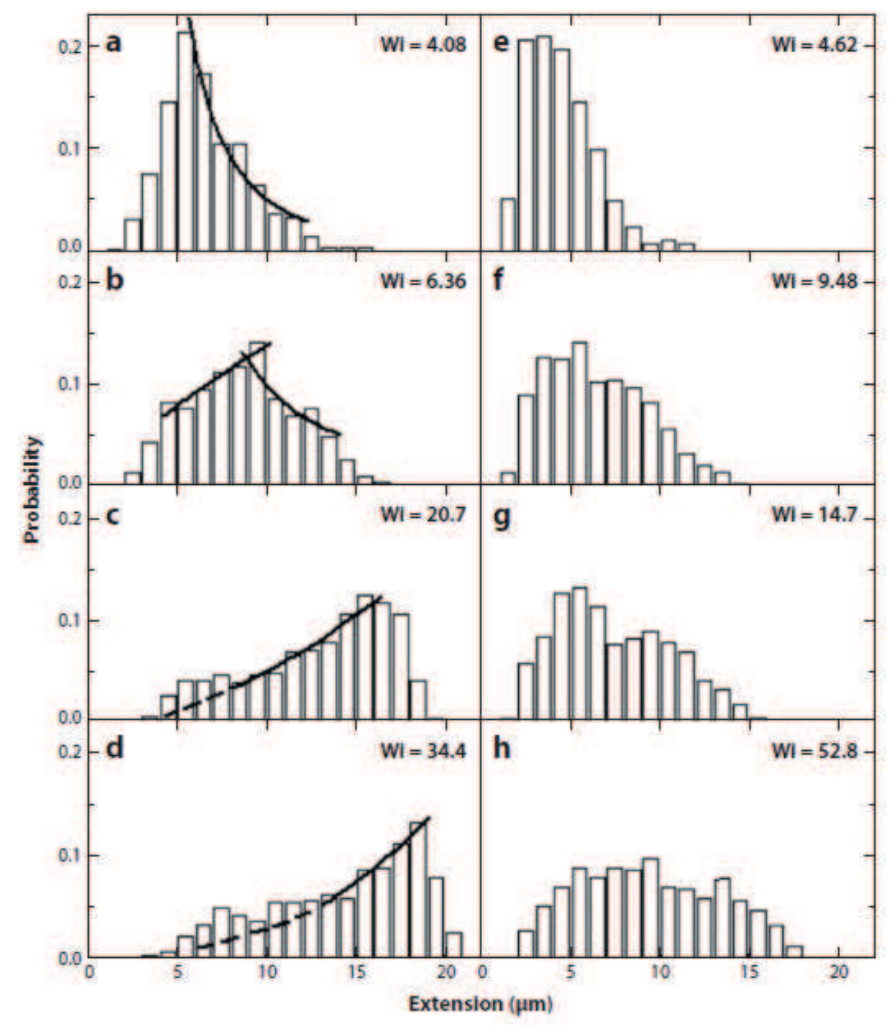

FIG. 1: Probability distribution $P(R)$ of the end-to-end polymer length $R=|\mathbf{R}|$ measured in a swirling flow. Black lines are best fit with power-law distribution $R^{-1-a}$ : A clear coil-stretch transition is observed for $\mathrm{Wi}=\mathrm{Wi}_{c} \sim 6$. For $\mathrm{Wi}<\mathrm{Wi}_{c}, P(R)$ shows a power-law distribution with $a \sim 1.5$ for the smallest Wi in panel (a). Adapted from Reference [12] with permission.

boundaries and review the ideas of Lumley [13] and de Gennes [14, 15] on when turbulence would be affected by polymers.

For three-dimensional homogeneous and isotropic turbulence, the Kolmogorov 1941 theory holds approximately with corrections due to intermittency. Energy is injected at the large scales, and cascaded down to smaller scales, and eventually dissipated at the Kolmogorov scale $\eta$ at which molecular viscosity directly acts. At each scale $r$ in the inertial range, there is a characteristic fluctuating velocity $\delta u(r)$, related to $r$ by

$$
\frac{[\delta u(r)]^{3}}{r} \approx \epsilon
$$

A crude estimate of the stretching at scale $r$ can be obtained by $\delta u(r) / r \equiv 1 / \tau_{r}$. Upon using Equation 5 we obtain $\tau_{r} \sim r^{2 / 3} \epsilon^{-1 / 3}$. Our discussions in Section 2.1 lead one to think that polymers would affect turbulence at scales $r$, where $\tau / \tau_{r} \geq 1$. Thus for scales $r \leq r^{*}$, where $r^{*}=\left(\epsilon \tau^{3}\right)^{1 / 2}$ is defined by $\tau_{r^{*}}=\tau$, turbulence would be affected in a certain way. This is the idea originally proposed by Lumley. According to this idea, polymers would affect the turbulent flow as long as $r^{*} \geq \eta$, regardless of the concentration of the polymers.

De Gennes followed a completely different approach. He thought that polymers could produce effect on turbulence only when the elastic energy stored by the polymers becomes comparable to the turbulent kinetic energy. He assumed that at scale $r$, the elastic energy of polymers per unit volume would decrease with $r$ as

$$
E_{e l}(r)=c k_{B} T\left(\frac{r^{*}}{r}\right)^{5 m / 2}
$$

where $c$ is the concentration of polymers per unit volume, $k_{B}$ is the Boltzmann constant, $T$ is the temperature of the polymer solution, and $1 \leq m \leq 2$ is some positive exponent depending on the specifics of the flow. Note that in Equation 1, the elastic energy is taken to be quadratic in $R$, i.e. $m=4 / 5$. The turbulent energy per unit volume at scale $r$ is given by $\rho[\delta u(r)]^{2}$, where $\rho$ is the density of the polymer solution and increases with $r$. Thus polymers would affect turbulence at scales $r \leq r^{* *}$, where the scale $r^{* *}$ is the scale at which the elastic energy and the turbulent 
kinetic energy become equal. Using Equations [5] and [6] and the expression for $r^{*}$, we obtain

$$
r^{* *}=\left(\frac{c k_{B} T}{\rho}\right)^{v} \epsilon^{1 / 2-v} \tau^{3 / 2-v},
$$

where $v=(5 m / 2+2 / 3)^{-1}$. For very small $c, r^{* *}$ is smaller than $\eta$, and polymers cannot affect turbulence. Thus there exists a threshold concentration for the polymers to have any effect on turbulence. For dilute polymer concentrations above the threshold value, $\eta<r^{* *}<r^{*}$ and as concentration increases $r^{* *}$ approaches $r^{*}$. De Gennes argued that polymers should be considered as passive for $r^{* *}<r<r^{*}$ and the turbulent energy cascade would stop at $r^{* *}$, so $r^{* *}$ can be thought of as an effective cutoff scale replacing the Kolmogorov scale $\eta$. However, he provided no information on how the turbulent energy is eventually dissipated.

Thus the theory of de Gennes allows us to see clearly how and why the concentration of polymers must play a role in their effects on turbulence, whereas Lumley's idea leads to the scale $r^{*}$, which is a possible upper-bound scale for polymers to affect turbulence. However, neither of the two theories gives any details on how turbulence is affected. Several experiments have been carried out to study the change in statistics of a turbulent counter-rotating disk flow by polymers [16 19]. Turbulence statistics such as Eulerian velocity structure functions are modified in the presence of polymers. It is found that the Eulerian velocity structure functions can be made to collapse into one master curve in Reference [18] but into two families, one for low polymer concentration and another for higher concentration in Reference [19], when the separation is rescaled by some length scale that depends on polymer concentration $c$ and relaxation time $\tau$. The precise form of this length scale is different in these experiments. Suggestions to relate this length scale to $r^{* *}$ or a new length scale at which the turbulent kinetic energy flux and the elastic energy flux are equal have been made [18]. However, we have yet to obtain a full understanding of all these experimental results.

\section{POLYMER EFFECT ON FLOWS: EXPERIMENTS}

\section{A. Drag Reduction}

One well-known effect is polymer reduces friction drag in turbulent wall-bounded flows. This effect was discovered by Toms [20], who observed that an addition of about 10 parts per million by weight of a long chain polymer (polymethyl methacrylate) can lead to a significant reduction of friction drag in a turbulent flow of monochlorobenzene in a pipe while studying the degradation of polymers. Similar effect has been observed in turbulent pipe or channel flow of water with polyethylene oxide or polyacrylamide. A large number of experimental studies have been carried out to characterize this phenomenon (see e.g. Reference [21] for a review). Below, we will summarize the key features of the phenomenon.

For a fluid of density $\rho$ flowing in a pipe of diameter $D$, the friction drag is measured by the dimensionless Fanning friction factor $f_{D}$, defined as the ratio between wall shear stress $\tau_{w}$, which is the work done per unit volume due to the applied pressure gradient, and the kinetic energy density of the mean flow

$$
f_{D} \equiv \frac{\tau_{w}}{\rho U_{a v}^{2} / 2}=\frac{D \Delta p}{2 \rho U_{a v}^{2} L} .
$$

Here $\Delta p$ is the pressure drop across a distance $L$ in the pipe, and $U_{a v}$ is the mean velocity, averaged over time and the cross-section of the pipe, and is given by $\int_{0}^{D / 2} V(y) 2 \pi(D / 2-y) d y /\left(\pi D^{2} / 4\right)$, where $V(y)$ is the streamwise velocity averaged over time and $y$ is the distance from the wall. When polymers are added, $f_{D}$ is not changed at low Reynolds number $\operatorname{Re}=U_{a v} D / \nu$. At a certain value $\operatorname{Re}_{c}$, the onset of drag reduction occurs and $f_{D}$ is reduced. Experimental measurements show that the onset value $\operatorname{Re}_{c}$ depends on polymer concentration [22]. The reduction of $f_{D}$ is tantamount to an increase in the mean streamwise velocity $V(y)$ for a given pressure drop $\Delta p$ or a decrease in the pressure drop required for a fixed mean streamwise velocity. Such an enhancement of $V(y)$ by polymers is shown in Figure 2 in terms of the commonly used wall units, defined by $V^{+}(y)=V(y) / u_{\tau}$ and $y^{+}=y u_{\tau} / \nu$, where $u_{\tau}=\sqrt{\tau_{w} / \rho}$ is the friction velocity. After the onset, $f_{D}$ decreases when the polymer concentration is increased, and $V^{+}(y)$ approaches an apparently universal limiting curve that cannot be exceeded by increasing the concentration further. This limit is referred to as the maximum drag reduction (MDR) asymptote (see Figure $2 \mathrm{a}$ ). Theories of drag reduction will be discussed in section 5.2 .

\section{B. Elastic Turbulence}

Another interesting effect is that polymer with sufficiently long relaxation time or high Weissenberg number Wi can give rise to an irregular flow state with velocity fluctuations spanning a broad range of spatial and temporal scales 
even at low Reynolds number. This irregular flow state at high Wi and low Re is known as elastic turbulence 23] and is caused by an instability due to the polymer stresses. The first experiments of elastic turbulence were performed in dilute polymer solution in flows with curved streamlines such as swirling flow between two plates, Taylor-Couette flow, and flow in a curvilinear channel [24]. More recently, elastic instability has also been observed experimentally in a long, straight microchannel [25].

For example, in a swirling flow between two plates, the fluid in a stationary cylindrical cup of flat bottom plate is set into swirling motion by a co-axial rotating upper plate just touching the surface of the fluid. The average shear stress $\sigma$ is measured as a function of the average shear rate $\dot{\gamma}$. For small Weissenberg number, defined by $\mathrm{Wi}=\tau \dot{\gamma}, \sigma$ remains close to the value $\sigma_{\text {lam }}=\eta(\dot{\gamma}) \dot{\gamma}$ for a laminar shear flow at the same angular velocity $\omega$, where $\eta(\dot{\gamma})$ is the viscosity of the polymer solution. It is found that when Wi is above some critical value, $\sigma / \sigma_{\text {lam }}$ increases significantly and there is a transition from the laminar flow to elastic turbulence. For the same range of shear flow rates of the pure solvent without polymers, the ratio $\sigma / \sigma_{\text {lam }}$ remains at unity within resolution of the measurements. The frequency power spectrum of the velocity fluctuations in elastic turbulence displays a power-law decay, which spans over about a decade in frequencies. The power-law dependence indicates a broad range of time scales of the motion and resembles that of developed turbulence of Newtonian fluid at high Re but the energy spectrum displays a steeper slope indicating that the flow is random but not as "rough" as in the case of fully developed turbulence of Newtonian fluid. The fluctuating velocity field can be visualized in the snapshots of the flow of the polymer solution above the transition as shown in Figure $2 \mathrm{~b}$.
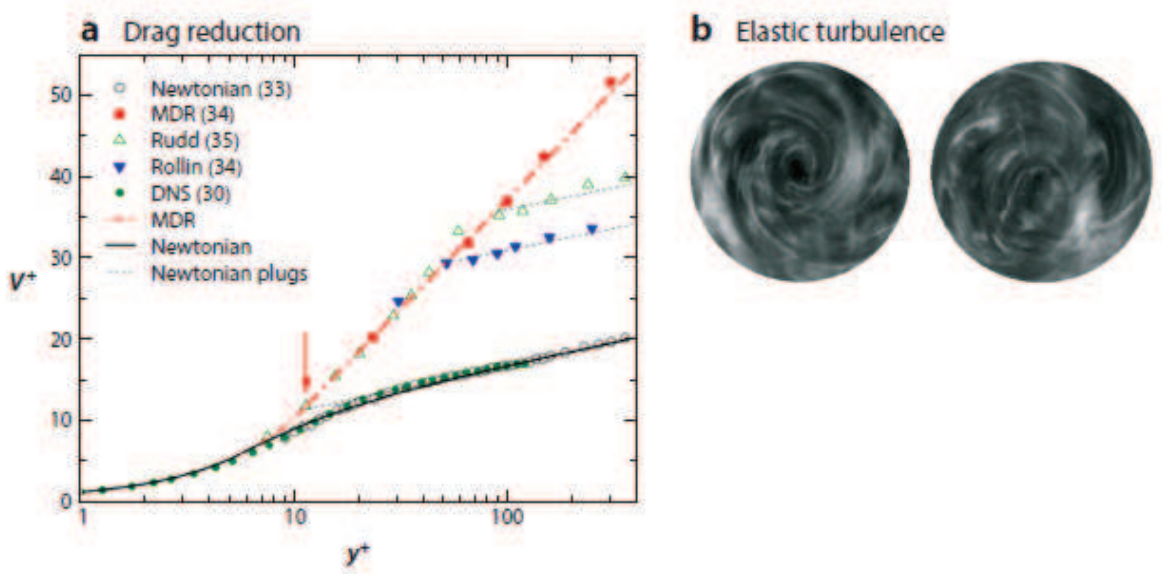

FIG. 2: (a) A synthetic view of experimental and numerical results on drag reduction in wall bounded turbulence. The horizontal axis represents in log scale the value of $y^{+}$, whereas the vertical axis shows the mean streamwise velocity $V^{+}$in wall units. The black solid line represents the Newtonian profile. The red dot-dashed line represents the MDR asymptote (see also [21]) and is a representation of Equation 26] By increasing the polymer concentration for fixed pressure drop, there is a systematic increase of drag reduction up to the asymptotic MDR regime. Reprodiced from Reference [39] with permission. (b) Elastic turbulence. Two representative snapshots of the polymer flow at $\mathrm{Wi}=13$ and $\mathrm{Re}=0.7$ taken from below. The field of view corresponds to the upper plate area. The flow was visualized by seeding the fluid with light reflecting flakes. Reproduced from Reference [23] with permission. Abbreviations: DNS, direct numerical simulations; MDR, maximum drag reduction.

\section{EQUATION OF MOTION}

Given Equation 2 for the conformational tensor $R_{i j}$, the effect of polymers on the flow can be computed using the momentum balance, which reads as

$$
\begin{aligned}
\partial_{t} u_{i}+u_{j} \partial_{j} u_{i} & =-\frac{1}{\rho} \partial_{i} p+\partial_{j} T_{i j}+\nu_{s} \Delta u_{i} \\
T_{i j} & \equiv \frac{\nu_{p}}{\tau}\left[f(R) \frac{R_{i j}}{R_{0}^{2}}-\delta_{i j}\right]
\end{aligned}
$$

Here, $\nu_{s}$ is the kinematic viscosity of the solvent and $\overline{\overline{\mathbf{T}}}$ (with components $T_{i j}$ ) represents the momentum stress tensor due to polymer, where $\nu_{p}$ is the polymer contribution to the zero-shear viscosity $\nu_{0}=\nu_{s}+\nu_{p}$ of the polymer solution 
and $\nu_{p}$ increases with polymer concentration $c$ [1]. Equation 9 is often presented by writing $\nu_{s}=(1-\beta) \nu_{0}$ and $\nu_{p}=\beta \nu_{0}$, which is convenient for numerical studies with a constant $\nu_{0}$. The overall free energy $F$ in the system is defined as

$$
F=\int d \mathbf{r}\left\{\frac{\rho u^{2}}{2}+\frac{\nu_{p}}{\tau}\left[\operatorname{tr} R_{i j}-R_{0}^{2} \ln \operatorname{det}\left(\overline{\overline{\mathbf{R}}} / R_{0}^{2}\right)\right]\right\}
$$

Using Equations 2 and 10, one can obtain the energy dissipation,

$$
\frac{d F}{d t}=\int d \mathbf{r}\left[\left\{\frac{\nu_{s}}{2}(\nabla u)^{2}+\frac{1}{\tau} \operatorname{tr}\left[\overline{\overline{\mathbf{T}}}(1+\overline{\overline{\mathbf{T}}})^{-1} \overline{\overline{\mathbf{T}}}\right]\right\}\right.
$$

Equations 2 and 9 with 10 are known as the finite extensible nonlinear elastic model with Peterlin's approximation, or the FENE-P model in short, for dilute polymer solutions. The FENE-P model is based on the assumption of a simple nonlinear elastic force describing polymer stretching whereas more complex physical effects are not taken into account (see, for instance Reference [26]) and it is always challenging to compare the relaxation time in the FENE-P model against real experiments. Nevertheless the FENE-P model can qualitatively capture the relevant experimental observations described in the previous section, namely elastic turbulence and drag reduction. If one ignores the finite extensibility of the polymer, $f(R)=1$ and the resulting equations are usually referred to as Oldroyd-B model. In the past few years, there have been a number of direct numerical simulations (DNSs) of the FENE-P or Oldroyd-B model for wall bounded turbulence [27 31] (see also 32] for a detailed review). In Figure 3 a, we show the numerical results obtained in [27] for the stream wise velocity profile for different values of Weissenberg number (here denoted by We). There is a clear increase in the velocity or a decrease in drag as We increases. The numerical results should be compared against the laboratory measurements shown in Figure 2 a. This is clear evidence that the FENE-P or Oldroyd-B model captures the qualitative behavior of drag reduction found in laboratory, although a quantitative agreement may be available only after fine tuning of the model parameters. Two-dimensional numerical simulations have been studied in References [36, 37] for a Kolmogorov flow with periodic boundary conditions. The system is forced with a periodic forcing and attains a velocity field $\left(U_{0} \cos (2 \pi y / L), 0\right)$ where $L$ is the size of the system. It is known that for $\operatorname{Re} \equiv U_{0} L / \nu_{0}$ smaller than the critical value $\operatorname{Re}_{c}=\sqrt{2}$, the flow is stable when there are no polymers. By increasing the Weissenberg number $\mathrm{Wi}=\tau U_{0} / L$, the system develops an instability and the dynamics becomes chaotic in time and space, i.e. the system shows the characteristic features of elastic turbulence. Figure 3 b, as well as a more detail analysis given in Reference [37], clearly shows that elastic turbulence can be explained (at least qualitatively) by the constitutive Equations (2) and (9]).

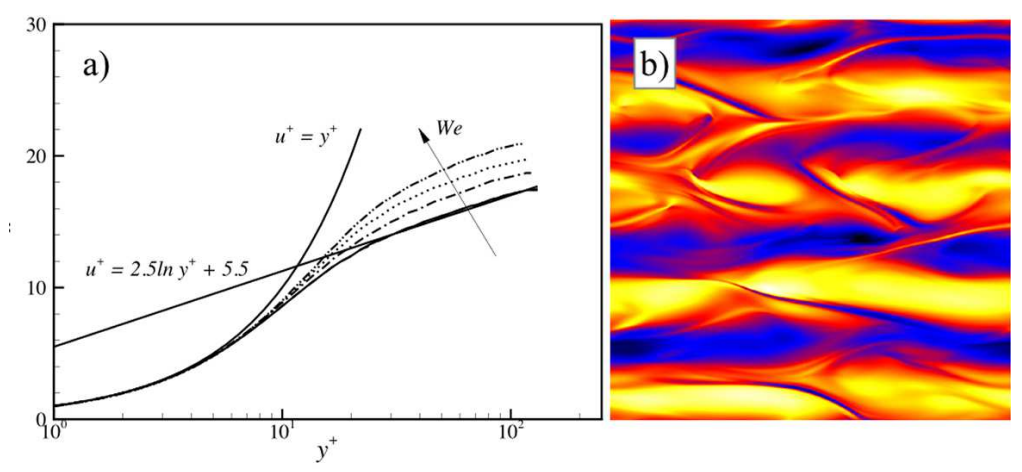

FIG. 3: (a) Velocity profile of the streamwise velocity $V^{+}$in Prandtl units, obtained in DNS of the Oldroyd-B model for different values of Weissenberg number, (here denoted by We); We $=\tau S_{0}$, where $S_{0}$ is the value of the shear at the boundary $y^{+}=0$. By increasing $\tau$ or We, one observes a clear decrease in the overall drag. Reproduced from Reference [27] with permission. (b) Snapshot of the vorticity field obtained in a two-dimensional simulation of Kolmogorov flow with polymer at $\mathrm{Re}=0.48$ and $\mathrm{Wi}=31$. The system shows a clear instability leading to a turbulent-like behavior similar to the phenomenon of elastic turbulence. Adapted from Reference [37] with permission.

There is a simple but highly non-trivial argument that allows us to understand the role of the concentration parameter $\nu_{p}$ in the FENE-P model [38, 39]. We consider the case where there is substantial stretching such that $\delta_{i j}$ in Equation [10 can be neglected. Let us assume that the concentration changes as $\nu_{p} \rightarrow n \nu_{p}$ where $n$ is any positive real number. This is equivalent to saying that $R_{i j} \rightarrow R_{i j} / n$ and, more importantly, the function $f(R)$ changes as

$$
f(R) \rightarrow \frac{1}{1-\left(\alpha / n^{2}\right) R^{2}}
$$


which shows that by changing the concentration we (formally) change the value of the maximum polymer extension $R_{\max }$ in the FENE-P equations. Next, we observe that it is possible to define an effective polymer relaxation time

$$
\tau_{\text {eff }} \equiv \frac{\tau}{f(R)},
$$

which gives

$$
\tau_{\text {eff }}(n)=\tau\left(1-\frac{\alpha}{n^{2}} R^{2}\right)
$$

Equation 15 tells us two pieces of information: (a) For $n \gg 1$ we obtain $\tau_{\text {eff }}=\tau$, i.e. for high concentration the FENE-P model becomes independent of the concentration itself; (b) for $n \ll 1$ even a very small amount of stretching leads to $\tau_{\text {eff }} \rightarrow 0$. In Section 2.1, we see that polymer stretching occurs for $\mathrm{Wi}_{\lambda}=\lambda \tau \sim 1$, thus we can safely assume that the effect of polymers on the flow becomes relevant when $\mathrm{Wi}_{\text {eff }} \equiv \tau_{\text {eff }}(n) \lambda$ is large enough. This is equivalent to say that there exists a critical value $n_{c}$ or a critical concentration, for which $\tau_{\text {eff }}\left(n_{c}\right) \lambda \sim 1$ : For $n<n_{c}$ or concentration below the critical value, the effect of polymers is negligible, whereas for $n>n_{c}$ polymers can change the (turbulent) properties of the flow. In other words, the FENE-P model seems to capture the basic argument due to de Gennes on the existence of a critical concentration for polymer effects on flows, without assuming any critical scale and/or any cascade argument for the energy flux. As a side result, for the Oldroyd-B model with $f(R)=1$, the effect of the concentration disappears and any change in the concentration is equivalent to a redefinition of $R_{i j}$ with no effect on the dynamics; the only relevant parameter being the polymer relaxation time $\tau$. This implies that changing the polymer relaxation time $\tau$ in Oldroyd-B model is qualitatively equivalent to changing concentration parameter $\nu_{p}$ for fixed $\tau$ in the FENE-P model. The above discussion is independent of the way turbulence is generated and, in particular, of any wall effect.

\section{DRAG REDUCTION}

\section{A. Drag reduction without drag}
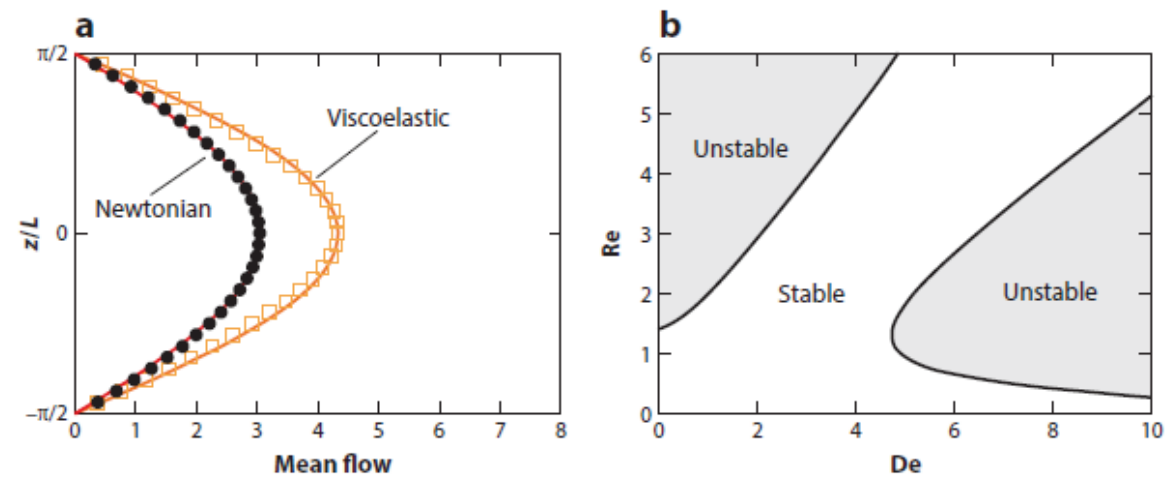

FIG. 4: (a) Comparison between the Newtonian profile and the Oldroyd-B simulations for a Kolmogorov flow with periodic forcing and mean flow $U \cos (z / L)$ with $\mathrm{De}=U \tau / L \sim 4$. A clear increase in the mean flow is observed for the polymeric case. Adapted from Reference [40] with permission. (b) Theoretical computation of the stability region in the plane Re-De for the Kolmogorov flows. Two different regions are observed: At small De the critical Reynolds number Re $\mathrm{e}_{c}$ increases for increasing De, i.e. the effect of polymers stabilizes the onset of hydrodynamical instability; at large De, $\mathrm{Re}_{c}$ decreases, i.e. instabilities are driven by polymer-flow interaction (elastic turbulence). Adapted from Reference [42] with permission.

The basic feature of fully developed three-dimensional turbulence is the Re-independence of the energy dissipation rate $\epsilon$. This feature, sometimes referred to as the zeroth law of turbulence, is often written as

$$
\epsilon=C_{D} \frac{U^{3}}{L}
$$

where $U$ represents the typical turbulent velocity and $L$ its corresponding scale. Notwithstanding the arbitrary definition of $U$ and $L$, the constant $C_{D}$ has the physical meaning of the drag coefficient. To see that this is the case, 
we can compute $\epsilon$ as $F U / \rho$ where $F$ is the forcing per unit volume acting on the system. From Equation 16 we obtain:

$$
C_{D}=\frac{F L}{\rho U^{2}}
$$

which is precisely the definition of the friction factor in wall-bounded turbulence (see Equation 8). Using Equation 16 and/or Equation [17, one can study the phenomenon of drag reduction for systems with no boundaries. Here we show some of the many cases studied in recent years. We start by reviewing the results obtained for three dimensional turbulent flow for a Kolmogorov forcing with periodic boundary conditions and mean flow $U \cos (z / L)$. We considered the limit of large $\operatorname{Re}=U L / \nu$, and relatively small $\mathrm{De}=U \tau / L$. In Figure 4 a, we show the main result obtained in [40] for Re $\sim 200$ and De $\sim 4$. The comparison between the Newtonian case (De $=0$ ) and the effect of polymers (De $\sim 4$ ) clearly shows that there is an increase of the mean flow due to polymers. A direct computation of $C_{D}$ given by Equation 17 shows a significant amount of drag reduction by almost a factor of two. Similar results have been obtained for turbulent flows forced by constant shear [41]. Following Reference [42], the case of Kolmogorov forcing can be studied analytically for small Re and De. The stability diagram for the Oldroyd-B model is displayed in Figure 4b. Two clear regions are present: For small Re and De, the critical value $R_{c}$ of the Reynolds number increases with increasing De, i.e., the flow is more stable due to the effect of polymers; for small Re and large De a second region of unstable flows appears and corresponds to elastic turbulence discussed in Section 3.2. Using multiple-scale analysis, it is possible to compute analytically the drag coefficient $C_{D}$ for relatively small Re and De (see Reference [43]). One obtains $C_{D}(\mathrm{De})=C_{D}(0) /(1+b \mathrm{De})$ with $b$ a positive constant, i.e. drag tends to decrease as De is increased. Although the above analytical results do not refer to turbulent flows, they clearly show that the phenomenon of drag reduction should be considered in more general terms and not only restricted to wall-bounded turbulent flows. The computation of the drag coefficient can also be done for homogeneous and isotropic turbulence. In Reference [44], DNSs of the FENE-P model have been performed for different values of De. In Table \ we show the value of $C_{D}$ (rightmost column) as a function of De (first column): Clearly for large enough De, we observe the effect of drag reduction (in the way defined in this section). To perform a scale by scale analysis for homogeneous and isotropic turbulence with polymers [44], we consider $R_{i j}=B_{i} B_{j}$, where $B_{i}$ is a vector field [3]. The equation for $B_{i}$ and $u_{i}$ are given by

$$
\begin{aligned}
\partial_{t} u_{i}+u_{j} \partial_{j} u_{i} & =-\frac{\partial_{i} p}{\rho}+\frac{\nu_{p}}{\tau} \partial_{j}\left[f(B) B_{i} B_{j}\right]+\nu_{s} \Delta u_{i}, \\
\partial_{t} B_{i}+u_{j} \partial_{j} B_{i} & =-\frac{1}{\tau}\left[f(B) B_{i}-1\right]+B_{j} \partial_{j} u_{i},
\end{aligned}
$$

where $f(B)=1 /\left(1-\alpha B_{i} B_{i}\right)$ with the repeated index summation notation used. The relative advantage in using Equations 18 and 19 is that we can easily compute the analog of the $4 / 5$ equation in this case, namely:

$$
\left\langle\delta u(r)^{3}\right\rangle+\frac{\nu_{p} f(B)}{\tau}\left\langle\delta u(r) \delta B(r)^{2}\right\rangle=-\frac{4}{5} \epsilon r-\frac{\nu_{p} f(B)}{\tau^{2}} \int_{r} d r^{\prime}\left\langle\delta B\left(r^{\prime}\right)^{2}\right\rangle,
$$

where $\delta u(r)=(\mathbf{u}(\mathbf{r}+\mathbf{x}, t)-\mathbf{u}(\mathbf{x}, t)) \cdot \mathbf{r} / r, \delta B(r)=(\mathbf{B}(\mathbf{r}+\mathbf{x}, t)-\mathbf{B}(\mathbf{x}, t)) \cdot \mathbf{r} / r$ and $r=|\mathbf{r}|$. We identify the de Gennes length scale $r^{* *}$ as $l_{p}$, the value of $r$ at which the two terms on the left-hand side of Equation 20 are equal. Similarly we can define the scale $r_{e}$ as the value of $r$ at which the two terms on the right-hand side are equal. If $r_{e} \sim l_{p}$, we obtain

$$
\frac{\delta u\left(l_{p}\right)}{l_{p}} \sim \frac{1}{\tau_{\text {eff }}}
$$

where we have used Equation 5. In other words the de Gennes scale $l_{p}$ satisfies the generalised Lumley criteria given by Equation 21. Using DNS [44] it is possible to show that $r_{e} \sim l_{p}$, as reported in Table [. The overall picture is that, within the FENE-P model, there exists a unique scale $l_{p}$ satisfying both the de Gennes criterion and the generalized Lumley criterion Equation 21. Hereafter, we shall refer to the scale $l_{p}$ as the Lumley-de Gennes scale. As discussed in the previous section, $l_{p}$ depends on the concentration. We remark that something similar to the scale $l_{p}$ has been extracted from experimental data in Reference [18] as discussed in Section 2.2.

Equations 18 and 19 can be used to define a shell model [38, 45 47]. It is worth noting that shell models have been extensively used to successfully understand some features of the statistical properties of intermittency in three dimensional turbulence. In this case, shell models are able to reproduce the relevant statistical features of DNS of homogenous and isotropic turbulence for FENE-P model, including drag reduction as previously defined. Also shell models can be used to demonstrate the existence of elastic turbulence at extremely low Re, as recently shown in [48] (see Figure 5a. In short, shells model can be used as a simple numerical tool to understand the basic features of energy fluxes end energy exchange in the FENE-P model and provide a clear cut definition for the Lumley-de Gennes scale $l_{p}$. 
TABLE I: Parameters of the numerical simulations discussed in [4]

\begin{tabular}{l|c|c|c|c}
\hline $\mathrm{De}$ & $\epsilon_{T}$ & $r_{e}$ & $l_{p}$ & $C_{D}=\epsilon_{T} /\left(u_{r m s}^{3} / L\right)$ \\
\hline 0 & 0.156 & - & - & 1.87 \\
0.18 & 0.174 & 1.23 & 0.603 & 1.90 \\
0.54 & 0.238 & 2.20 & 0.973 & 1.55 \\
0.54 & 0.232 & 2.20 & 0.923 & 1.60 \\
\hline
\end{tabular}
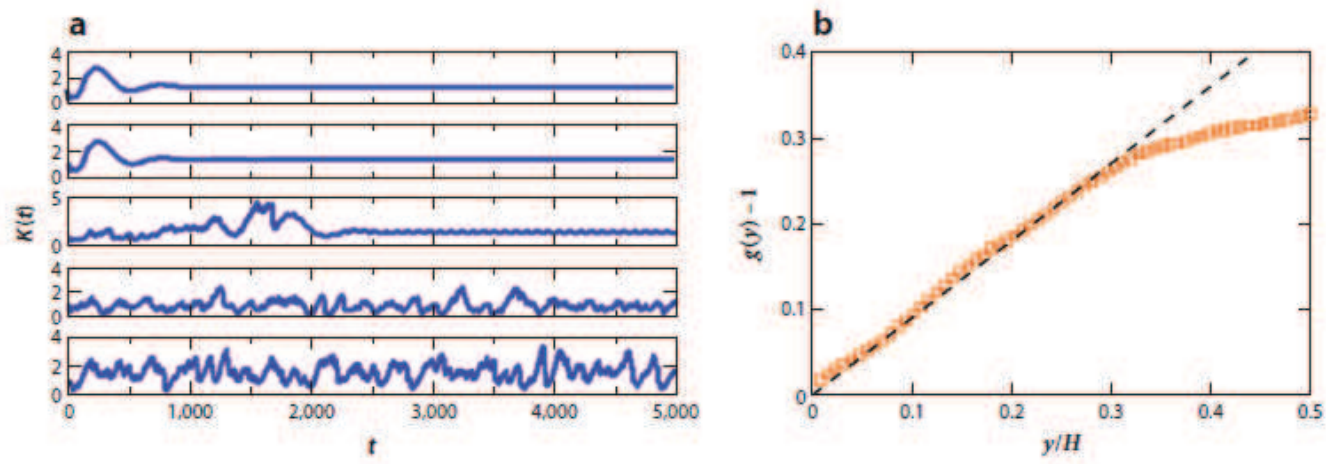

FIG. 5: (a) Elastic turbulence in shell models. The Weissenberg number Wi is increasing from the top to the bottom: At low Wi the model is not chaotic, whereas by increasing Wi the model exhibits chaotic behavior due to polymer-flow interaction. Adapted from Reference [48] with permission. (b) Computation of the effective viscosity $g\left(y^{+}\right)$in wall-bounded turbulence. The effective viscosity is computed as the ratio of energy dissipation rate due to polymer and energy dissipation rate due to turbulent fluctuations: $g\left(y^{+}\right)-1$ exhibits a linear profile (dashed line) close to the wall. Panel b courtesy by A. Scagliarini.

\section{B. Drag reduction in wall bounded flows}

We now turn our attention on the phenomenon of drag reduction in wall-bounded turbulence. Our major interest is to understand how the average velocity profile changes due to polymer effects in turbulent flows, as described in section 2.1. Our starting point is to briefly review the von Karman theory for wall-bounded turbulence. Using wall units defined in section 3.1, we can define $H^{+} \equiv H u_{\tau} / \nu=\operatorname{Re}_{\tau} \gg 1, S^{+}=\partial_{y} V(y) \nu / u_{\tau}^{2}$ the dimensionless shear of the average flow $V^{+}$and $W^{+}=-\langle w u\rangle / u_{\tau}^{2}$ the (absolute) turbulent momentum flux. Then momentum conservation reads:

$$
S^{+}+W^{+}=1-\frac{y^{+}}{\operatorname{Re}_{\tau}}
$$

Hereafter we shall neglect the last term on the right-hand side of Equation 22 To close the problem, we need a relation between $S^{+}$and $W^{+}$in agreement to the von Karman hypothesis. It turns out [39] that such a phenomenological equation can be derived using the energy balance in the form of

$$
\left[\frac{\delta^{+}}{y^{+}}\right]^{2}+\frac{\sqrt{W^{+}}}{\kappa_{v} y^{+}}=S^{+}
$$

Upon multiplying Equation 23 by the turbulent kinetic energy $K^{+} \sim W^{+}$, we can interpret as follows: the first term on left-hand side is the turbulent dissipation near the viscous layer $y^{+}=\delta^{+} \equiv \nu / u_{\tau}$; the second term on the left-hand side is the turbulent energy dissipation obtained by von Karman original argument while the term on the right-hand side is the turbulent energy production. Solution of Equations 22 and 23 gives the Newtonian profile (black line) shown in Figure 2 panel (b) with $\delta^{+}=5.7$ and $\kappa_{v}=0.4$. Asymptotically, we have $V\left(y^{+}\right)=\left(u_{\tau} / \kappa_{v}\right) \log \left(y^{+}\right)+A$ where $A \sim 5.5$. The region between the asymptotic behavior of $V\left(y^{+}\right)$and $\delta^{+}$is usually referred to as the buffer layer, i.e., a region near the wall where the maximum of turbulent energy production is located.

Lumley [13] and de Gennes [15] provided different theoretical interpretations for drag reduction in wall-bounded turbulent flows ( see also 32] for a recent review). Lumley attributed drag reduction to an increase of the buffer layer: "The conclusion is that, granted the law of the wall and the defect law, a drag reduction must appear as a 
thickened sublayer... The sensitivity is such that doubling the sublayer thickness about halves the skin friction" ([13], p. 376). According to Lumley, the increase of the buffer layer is due to an overall increase in the fluid viscosity because of polymer stretching and there should exist a critical polymer relaxation time $\tau$ for drag reduction to occur. He also argued, without any quantitative derivations, that the increase should be proportional to polymer concentration for small concentration. At variance with Lumley's interpretation, de Gennes suggested that polymers changes the Kolmogorov cascade of turbulence at a critical scale where most of the turbulent energy goes to polymer elastic stretching. The de Gennes scale depends on polymer concentration and there should exist a critical concentration for drag reduction to occur. Nevertheless de Gennes argued: "But the net result is still an enhancement of the intermediate 'buffer layer'. We expect drag reduction from this, although we have not carried out detailed analog Lumley' matching" ([15], p. 35). It is worthwhile to mention that de Gennes argued that the critical scale for polymer stretching should act as effective cutoff scale for turbulent fluctuations, i.e., an effective Kolmogorov scale, although de Gennes stated "Thus we do not know the ultimate fate of the turbulent energy" ([15], p. 44). Finally, de Gennes never mentioned drag reduction as induced by an energy flux that went from small scale polymer fluctuations to turbulent fluctuations. At any rate, neither de Gennes nor Lumley was able to provide the explanation of why the buffer layer should increase due to polymer stretching. Also, at the time when Lumley and de Gennes theories were proposed, no DNSs based on the FENE-P or Oldroyd-B models were available. Our task is now to provide a quantitative explanation for drag reduction and MDR within the framework of the FENE-P or Oldroyd-B models, in which the ultimate fate of turbulent kinetic energy, i.e., the energy dissipation, is given by Equation 12 . One possible answer to our question is provided in References [39] and [49], and it is based on the idea that the polymer stretching produces an effective space-dependent viscosity. The phenomenological theory is based on the following observations:

1. In wall-bounded turbulent flows, where the local shear $S$ is large, the polymer conformational tensor satisfies the inequalities $R_{x x} \gg R_{x y} \gg R_{y y} \sim R_{z z}$.

2. To first order approximation one can estimate $R_{x x} \sim S \tau_{\text {eff }} R_{x y}$ and $R_{x y} \sim S \tau_{\text {eff }} R_{y y}$ where $\tau_{\text {eff }}$ is given in Equation 14.

3. Turbulent kinetic energy $K(y)$ is fixed by the Lumley-de Gennes criteria $\sqrt{K} \sim y / \tau_{\text {eff }}$.

4. Turbulent energy dissipation is the sum of the viscous effect and $\nu_{p}\left(R_{y y}+R_{z z}\right) / \tau_{\text {eff }}^{2} \sim \nu_{p} K(y) R_{y y} / y^{2}$.

Point 2 implies that that terms $\left\langle R_{x k} \partial_{k} u_{x}^{\prime}\right\rangle$ can be neglected, where $u_{x}^{\prime}$ is the turbulent velocity fluctuation in $x$ direction. Based upon the previous points, one can show that Equations 22 and 23 are modified as

$$
\begin{array}{r}
\nu^{+}\left(y^{+}\right) S^{+}+W^{+}=1 \\
\nu^{+}\left(y^{+}\right)\left(\frac{\Delta^{+}}{y^{+}}\right)^{2}+\frac{\sqrt{W^{+}}}{\kappa_{v} y^{+}}=S^{+}
\end{array}
$$

where $\nu^{+}=1+A R_{y y}$, and $A$ is a constant depending on the ratio $\nu_{p} / \nu_{s}$ and $f(R)$, and $\Delta^{+}$is a constant that approaches $\delta^{+}$in the limit concentration $c \rightarrow 0$. We remark that the very existence of a space-dependent viscosity can be numerically checked by computing the quantity $g(y)=\epsilon_{T} / \nu_{0}\left\langle(\nabla \mathbf{u})^{2}\right\rangle$, where $\epsilon_{T}$ is the total energy dissipation in the system and $\nu_{0}\left\langle(\nabla \mathbf{u})^{2}\right\rangle$ is the energy dissipation due to velocity fluctuations. It turns out that $g(y)-1$ is a linear function of $y$ with very good accuracy (see, for instance Figure 50 ). Also, the existence of a space-dependent viscosity can be derived in the generalization of the classical Prandtl-Blasius boundary layer theory for the Oldroyd-B or FENE-P models, as recently shown in References [50, 51] (see Section 6). The approach described in Reference [39] justifies two independent questions: (a) Does space-dependent viscosity produce drag reduction? (b) What is the functional form of $\nu^{+}\left(y^{+}\right)$that maximizes drag reduction and, eventually, provide a quantitative prediction of the MDR asymptote? In principle, both questions can be answered using numerical simulations. In Reference [52], linear viscosity profiles are shown to produce drag reduction in wall-bounded turbulent flows and, for increasing slope of the space-dependent viscosity, one observes a decrease in the drag. Concerning the second question, using Equations 24 and 25. Reference [53] showed that the MDR can be achieved when $\nu^{+}\left(y^{+}\right)$is linear and that the resulting velocity profile is given by

$$
V^{+}\left(y^{+}\right)=12 \ln \left(y^{+}\right)-17.8
$$

assuming $\delta^{+} \sim 6$. Equation 26 is plotted in Figure 2b and it is extremely close to the MDR behavior discussed in Reference [21]. It is interesting to remark that the asymptotic behavior given by Equation 26 is achieved in the limit $W^{+} \rightarrow 0$, i.e., in the limit turbulence tends to vanish. In this limit, therefore, the Lyapunov exponent of the system becomes extremely small and, consequently, the correlation time of any fluctuating quantity becomes very long. This phenomenon is observed in DNS of the FENE-P model as discussed in Reference [54], where periods of hibernation 
and activation of turbulence activity are observed near MDR. We emphasize that the formulation of the MDR as a variational problem for the space-dependent viscosity implies the universality of the MDR behavior, independent of polymer characteristics. For very large Wi there may be non-negligible contributions from correlations between the fluctuating strain rates $\partial_{i} u_{j}^{\prime}$ and the conformational tensor $R_{i j}$. These contributions may support turbulent fluctuations near the MDR asymptote in a way similar to the effect of elastic turbulence, i.e., there may be on average a flux of energy from polymer to turbulence. This possibility was pointed out in References [55] and [56] and referred to as elasto-inertial turbulence. However, this effect may be consistent with the existence of a space-dependent viscosity described in Equations 24 and 25. Also, for homogeneous shear flow simulations reported in [41] as well as in the numerical simulations of wall-bounded turbulence discussed in Reference [31], no energy flux from polymer to turbulence has been found.

It is worth noting that asymptotic behavior similar to MDR is observed in numerical simulations of the FENE-P model in homogeneous shear turbulence [41], where the concept of a space-dependent viscosity cannot work. However, the concept of space-dependent viscosity can be generalized to scale-dependent viscosity as discussed in Reference [57] and a scale-dependent viscosity may explain drag reduction for the cases reviewed in Section 5.1.

\section{POLYMER IN HEAT TRANSFER FLOWS}

The effect of polymers on heat transfer has been studied in turbulent Rayleigh-Bénard (RB) convection. In the RB system, a fluid is constrained between two horizontal plates that are heated from below and cooled from above, and the system is controlled by two parameters: the Rayleigh number, $\mathrm{Ra}=\alpha g \delta T H^{3} /\left(\kappa \nu_{s}\right)$, which measures the thermal forcing due to the temperature difference $\delta T$ between the two plates, and the Prandtl number, Pr $=\nu_{s} / \kappa$, which is the ratio between the kinematic viscosity $\nu_{s}$ and the thermal diffusivity $\kappa$ of the fluid. In addition, $\alpha$ is the isobaric volume expansion coefficient of the fluid, $g$ the acceleration due to gravity, and $H$ is the vertical distance between the top and bottom plates. In turbulent RB convection, there are distinct flow regions, namely viscous boundary layers near all rigid walls and two thermal boundary layers, one above the bottom plate and one below the top plate, and an approximately homogeneous bulk flow in the central region of the convection cell. In the Boussinesq approximation, the equations of motion for RB convection with polymers are

$$
\begin{aligned}
\partial_{t} u_{i}+u_{j} \partial_{j} u_{i} & =-\frac{1}{\rho} \partial_{i} p+\partial_{j} T_{i j}+\nu_{s} \Delta u_{i}+\alpha g\left(T-T_{*}\right) \delta_{i z} \\
\partial_{t} T+u_{j} \partial_{j} T & =\kappa \Delta T
\end{aligned}
$$

where $T$ is the temperature field, $T_{*}$ is the mean temperature averaged over time and the whole system, $z$ is along the vertical direction, and the polymer stress tensor $T_{i j}$ is given by Equation [10 with the conformation tensor $R_{i j}$ governed by Equation 2. Heat transport is measured by the Nusselt number (Nu), which is the normalized heat flux defined by

$$
\mathrm{Nu} \equiv \frac{\left\langle u_{z} T-\kappa \partial_{z} T\right\rangle_{A}}{\kappa \delta T / H}
$$

where $\langle\cdots\rangle_{A}$ denotes an average over a horizontal plane of the convection cell and time.

The bulk flow of turbulent RB convection without the boundary layers is believed [58] to be a good approximation of the ultimate regime at large Ra. The effect of polymer in this regime has been studied by DNS of the equations of motion with periodic boundary conditions [59]. In this case, polymer enhances the length scale $l_{T}$ of thermal plumes and heat transfer increases as $\mathrm{Nu} \sim l_{T}^{3 / 2} \sim \mathrm{Wi}^{3 / 2}$, where $\mathrm{Wi} \equiv \tau U_{c} / H$ and $U_{c}=\sqrt{\alpha g \delta T H}$. A similar enhancement in heat transfer by polymers has been found in Rayleigh-Taylor turbulence [60, 61] (see Figure 6). In contrast, an experimental investigation [62] of turbulent $\mathrm{RB}$ convection at moderate Ra reported a small but clear reduction of $\mathrm{Nu}$ in the presence of polymers, and the amount of heat reduction increases with polymer concentration. At low Ra, most of the energy dissipation is concentrated in the boundary layers, and thus the numerical results for the ultimate regime do not apply. In later experimental studies [63, 64, both a convection cell with usual smooth top and bottom plates and a convection cell with rough top and bottom plates have been used. The reduction of heat transport by polymers at moderate Ra has been confirmed in the smooth cell, whereas an enhancement in heat transfer is observed in the rough cell when the polymer concentration is not too small (see Figure 7). It is believed that the pyramidal structures of the rough plates perturb the boundary layers and make the flow resembling that of the bulk flow even at moderate Ra.

In an attempt to understand the experimental results at moderate Ra in the usual smooth convection cell, the effect of polymers in the framework of the classical Prandtl-Blasius boundary layer theory was analyzed [50, 51], which is known [65, 66] to be a good approximation for moderate Ra and stable boundary layers. The outcomes 


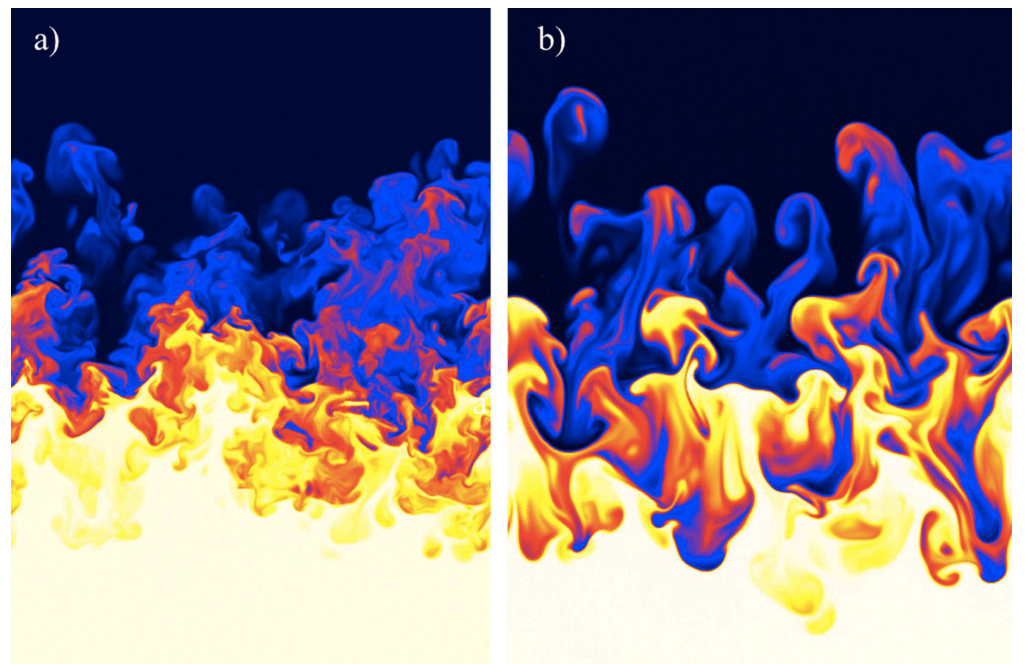

FIG. 6: Snapshots of the temperature field (a) without and (b) with polymers in direct numerical simulation of Rayleigh-Taylor turbulence. Due to polymers, the turbulent plumes become larger in size and small scales fluctuations are suppressed. As a result, the heat flux increases. Reproduced from Reference [60] with permission.
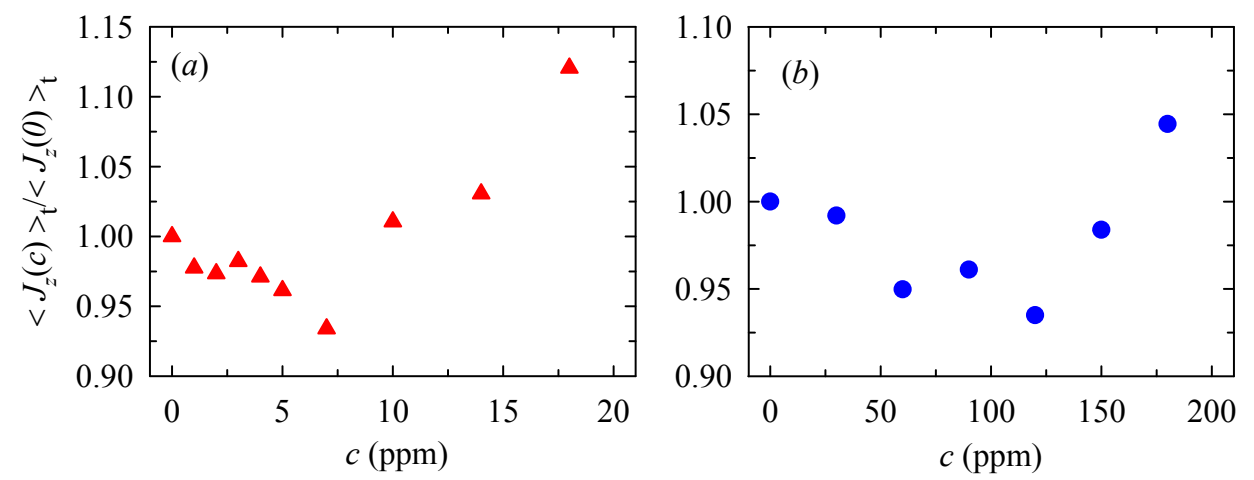

FIG. 7: Time-averaged local vertical heat flux $\left\langle J_{z}(c)\right\rangle_{t} \equiv\left\langle u_{z}(t)\left[T(t)-T_{*}\right]\right\rangle_{t} H /(\kappa \delta T)$ at the center of the rough convection cell, normalized by its Newtonian value $\left\langle J_{z}(0)\right\rangle_{t}$ measured at the same Ra, as a function of polymer concentration $c$ for (a) polyacrylamide and (b) polyethylene oxide. Adapted from Reference [64] with permission.

of the approach discussed in References [50, 51] are the following: First, the effect of polymers induces an effective space-dependent viscosity in the equation of motion, which peaked in the boundary layer and vanishes away from the boundary layer. Second, upon increasing the polymer concentration, heat reduction is observed, i.e., Nu decreases with respect to $\mathrm{Nu}_{0}$ corresponding to the Newtonian flow with no polymer at the same zero-shear viscosity. Finally, it can be shown that if the effective viscosity extends into the bulk of the system, i.e., the center of the cell, the value of $\mathrm{Nu}$ increases against $\mathrm{Nu}_{0}$. Physically this means that heat enhancement should be observed if a substantial stretching of the polymer in the bulk of the system exists. The last statement has been confirmed by a very recent DNS of turbulent RB convection with polymers [67] at moderate $\mathrm{Ra}$ and $2 \leq \mathrm{Wi} \leq 50$.

\section{CONCLUSIONS}

Polymer-flow interactions lead to a number of non-trivial and counterintuitive phenomena observed in laboratory experiments, namely drag reduction, elastic turbulence and modification of heat transport in natural convection. Our present knowledge is mostly based on direct numerical simulations, which provide a qualitative (and in some cases quantitative) explanation of experimental results. Still many different questions should be answered in order to define 
a possible unified scenario (if it exists). In this review, we have highlighted the most relevant results obtained in the last twenty years trying to capture the basic features underlying different theoretical approaches and explanations. It is fair to say that much progress have been made by suitable analyses of both laboratory and numerical investigations. Such progress has been able to foster new theoretical interpretations and to open a new view on the subject. The same approach can be applied to study the interaction of turbulent and/or laminar flows for other complex fluids as in the case of rigid polymers and surfactants. The outcomes of such investigations will be relevant for both our basic scientific understanding of complex fluid dynamics and technological applications.

\section{SUMMARY POINTS}

1. FENE-P and Oldroyd-B models can explain qualitatively most of the experimental results on drag reduction and elastic turbulence.

2. A clear cut definition of coil-stretch transition has been successfully provided in terms of Lagrangian properties of polymer dynamics.

3. The notion of drag reduction can be generalized to flows without boundaries.

4. Polymer-flow interaction can change the heat transport in natural and forced convection.

\section{FUTURE ISSUES}

1. More realistic models of flexible polymers are needed to make quantitative predictions of polymer-flow interactions.

2. Inertial effects on polymer dynamics, due to density mismatch between polymer and flow, need to be investigated.

\section{DISCLOSURE STATEMENT}

The authors are not aware of any affiliations, memberships, funding, or financial holdings that might be perceived as affecting the objectivity of this review.

\section{ACKNOWLEDGMENTS}

R.B. acknowledges funding from the European Research Council under the European Union's Seventh Framework Programme, AdG ERC Grant Agreement No. 339032 and E.S.C.C. acknowledges support from the Hong Kong Research Grants Council (Grants no 400304, 400311).

[1] Bird RB, Curtiss CF, Armstrong RC, Hassager O. 1987. Dynamics of Polymeric Fluids, Vol. 2, (New York, Wiley)

[2] Balkovsky E, Fouxon A, Lebedev V. 2000. Phys. Rev. Lett. 84:4765

[3] Balkovsky E, Fouxon A, Lebedev V. 2001. Phys. Rev. E 64:056301

[4] Chertkov M. 2000. Phys. Rev. Lett. 84:4761

[5] Celani A, Musacchio S, Vincenzi D. 2005. J. Stat. Phys. 118:531

[6] Benzi R, Paladin G, Parisi G, Vulpiani A. 1985. J. Phys. A:Math. Gen. 18:2157

[7] Boffetta G, Celani A, Musacchio S. 2003. Phys. Rev. Lett. 91:034501

[8] Martins Alfonso M, Vincenzi D. 2005. J. Fluid Mech. 540:99

[9] Musacchio S, Vincenzi D. 2011. J. Fluid Mech. 670:326

[10] Watanabe T, Gotoh T. 2010. Phys. Rev. E 81:066301

[11] Davoudi J, Schumacher J. 2006. Phys. Fluids 18:025103

[12] Gerashchenko S, Chevallard C, Steinberg V. 2005. Europhys. Lett. 71: 221

[13] Lumley JL. 1969. Annu. Rev. Fluid Mech. 1:367

[14] Tabor M, de Gennes PG. 1986. Europhys. Lett. 2:519

[15] De Gennes PG. 1990. Introduction to polymer dynamics (Cambridge University Press) 
[16] Crawford, AM, Mordant N, Xu H, Bodenschatz E. 2008. New J. Phys. 10:123015

[17] Ouellette NT, Xu H, Bodenschatz E. 2009. J. Fluid Mech. 629:375

[18] Xi H-D, Bodenschatz E, Xu H. 2013. Phys. Rev. Lett. 111:024501

[19] de Chaumont Quitry A, Ouellett NT. 2016. Phys. Rev. E 93:063116

[20] Toms BA. 1948. Proc. 1st Int. Congr. on Rheology 2:135

[21] Virk PS. 1975. AIChE J. 21:625

[22] Nadolink RH. 1987. Ph.D. thesis, University of California at San Diego, La Jolla, CA, USA

[23] Groisman A, Steinberg V. 2000, Nature 405:53

[24] Groisman A, Steinberg V. 2004. New J. Phy. 6:29

[25] Pan L, Morozov A, Wagner C, Arratia PE. 2013. Phys. Rev. Lett. 110:174502

[26] Celani A, Puliafito A, Vincenzi D. 2006. Phys. Rev. Lett. 97:118301

[27] Min T, Yoo JY, Choi H, Joseph DD. 2003. J. Fluid Mech. 486:213

[28] Sureshkumar R, Beris AN, Handler RA. 1997. Phys. Fluids 9:743

[29] Dimitropoulos CD, Sureshkumar R, Beris AN. 1998. J. Non-Newtonian Fluid Mech. 79:433

[30] De Angelis E, Casciola CM, L'vov VS, Piva P, Procaccia I. 2003. Phys. Rev. E 67:056312

[31] Ptasinski PK, Boersma BJ, Nieuwstadt FTM, Hulsen MA, van den Brule BHAA, Hunt JCR. 2003. J. Fluid Mech. 49:251

[32] White CM, Godfrey MG. 2008. Annu. Rev. Fluid Mech. 40:235

[33] Warholic MD, Massah H, Hanratty TJ. 1999. Expts. Fluids 27:461

[34] Rolin A, Seyer FA. 1972. Can. J. Chem. Eng. 50:714

[35] Rudd MJ. 1969. Nature 224:587

[36] Boffetta G, Celani A, Musacchio S. 2003. Phys. Rev. Lett. 91:034501

[37] Berti S, Bistagnino A, Boffetta G, Celani A, Musacchio S. 2008. Phys. Rev. E 77:055306(R)

[38] Benzi R, Ching ESC, Horesh N, Procaccia I. 2004. Phys. Rev. Lett. 92:078302

[39] Procaccia I, L'vov VS, Benzi R. 2008. Rev. Mod. Phys. 80:225

[40] Boffetta G, Celani A, Mazzino A. 2005. Phys. Rev. E 71:036307

[41] Ashish R, Vaithianathan T, Collins LR, Brasseur JG. 2010. J. Fluid Mech. 657:189.

[42] Boffetta G, Celani A, Mazzino A, Puliafito A, Vergassola M. 2005. J. Fluid Mech. 523:161

[43] Boffetta G, Bistagnino A, Celani A, Mazzino A, Puliafito A, Vergassola M. 2007. J. Fluid Mech. 590:61

[44] De Angelis E, Casiola CM, Benzi R, Piva R. 2005. J. Fluid Mech. 531:1

[45] Benzi R, De Angelis E, Govindarajan R, Procaccia I. 2003. Phys. Rev. E 68:016308

[46] Kalehar C, Govindarajan R, Pandit R. 2005. Phys. Rev. E 72:017301

[47] Benzi R, Horesh N, Procaccia I. 2004. Europhys. Lett. 68:2

[48] Ray SS, Vincenzi D. 2016. Europhys. Lett. 114:44001

[49] Benzi R, De Angelis E, L'vov VS, Procaccia I, Tiberkevich V. 2006. J. Fluid. Mech. 551:185

[50] Benzi R, Ching ESC, Chu VWS. 2012. J. Fluid. Mech. 696:330

[51] Benzi R, Ching ESC, Yu WCK, Wang Y. 2016. J. Fluid Mech. 788:337

[52] De Angelis E, Casciola CM, L'vov VS, Pomyalov A, Procaccia I, Tiberkevich V. 2004. Phys. Rev. E 70:055301(R)

[53] Benzi R, De Angelis E, L’vov VS, Procacca I. 2005. Phys. Rev. Lett. 95:194502

[54] Graham MD, Xi L. 2012. Phys. Rev. Lett. 108:028301

[55] Samanta D, Dubief Y, Holzner M, Schäfer C, Morozov AN, et al. 2013. Proc. Natl. Acad. Sci. 110:12498

[56] Dubief Y, Terrapon VE, Soria J. 2013. Phys. Fluids 25:110817

[57] Benzi R, Ching ESC, Procaccia I. 2004. Phys. Rev. E 70:026304

[58] Lohse D, Toschi F. 2003. Phys. Rev. Lett. 90:034502

[59] Benzi R, Ching ESC, De Angelis E. 2010. Phys. Rev. Lett. 104:024502

[60] Boffetta G, Mazzino A, Musacchio S, Vozella L. 2010. Phys. Rev. Lett. 104:184501

[61] Boffetta G, Mazzino A. 2017, Ann. Rev. Fluid Mech. 49:119

[62] Ahlers G, Nikolaenko A. 2010. Phys. Rev. Lett. 104:034503

[63] Wei P, Ni R, Xia K.-Q. 2012. Phys. Rev. E 86:016325

[64] Xie YC, Huang S.-D, Funfschilling D, Li X.-M, Ni R, Xia K.-Q. 2015. J. Fluid Mech. 784:R3

[65] Grossmann S, Lohse D. 2000. J. Fluid Mech. 407:27

[66] Ahlers G, Grossmann S, Lohse D. 2009. Rev. Mod. Phys. 81:503

[67] Benzi R, Ching ESC, De Angelis E. 2016. Phys. Rev. E 94:063110 